\title{
Logarithmic quasinormal modes of a spin-3 field around the BTZ black hole
}

\author{
Yong-Wan Kim ${ }^{1, a}$, Yun Soo Myung ${ }^{1, b}$, and Young-Jai Park ${ }^{2,3, c}$ \\ ${ }^{1}$ Institute of Basic Science and School of Computer Aided Science, \\ Inje University, Gimhae 621-749, Korea \\ ${ }^{2}$ Department of Physics and Center for Quantum Spacetime, \\ Sogang University, Seoul 121-742, Korea \\ ${ }^{3}$ Department of Global Service Management, \\ Sogang University, Seoul 121-742, Korea
}

\begin{abstract}
$\underline{\text { Abstract }}$
Using the operator approach, we obtain logarithmic quasinormal modes and frequencies of a traceless spin-3 field around the BTZ black hole at the critical point of the spin-3 topologically massive gravity. The logarithmic quasinormal frequencies are also confirmed by considering logarithmic conformal field theory.
\end{abstract}

PACS numbers: 04.70.Bw, 04.30.Nk, 04.60.Kz, 04.60.Rt

Keywords: Logarithmic quasinormal modes; BTZ black hole; spin-3 topologically massive gravity

${ }^{a}$ ywkim65@gmail.com

bysmyung@inje.ac.kr

cyjpark@sogang.ac.kr 


\section{Introduction}

Recently, higher-spin theories on the $(2+1)$-dimensional anti-de Sitter $\left(\mathrm{AdS}_{3}\right)$ spacetimes have been the subject of active interest because they admit a truncation to an arbitrary maximal spin $N$ [1, 2]. Especially, the prototype of spin-3 model is a totally symmetric third-rank tensor of spin-3 field coupled to topologically massive gravity (TMG). Chen et al., 3] have developed the methods obtaining quasinormal modes of arbitrary spin theories, and discussed the traceless spin-3 fluctuations around the $\mathrm{AdS}_{3}$ spacetimes. They found that there exists a single massive propagating mode, besides left-moving and right-moving massless modes (gauge artifacts). On the other hand, Bagchi et al., [4] have independently studied the spin-3 TMG. They showed that the trace modes carry energy opposite in sign to the traceless modes, and pointed out the instability in the bulk of the logarithmic partner of the traceless modes. These are considered through extended analysis of spin-2 field in the cosmological TMG [5].

Very recently, Datta and David [6] have introduced massive wave equations of arbitrary integer spin fields including spin-3 fields in the Bañados-Teitelboim-Zanelli (BTZ) black hole background. Then, they have obtained their quasinormal modes that are consistent with the location of the poles of the corresponding two-point function in the dual conformal field theory. This could be predicted by the $\mathrm{AdS}_{3} / \mathrm{CFT}_{2}$ correspondence. By the way, they have solved the second-order perturbed equation of $\left[\square-m^{2}+4 / \ell^{2}\right] \Phi_{\rho \mu \nu}=0$ for spin-3 field with the ingoing modes at horizon and Dirichlet boundary condition at infinity. However, in this case, there exists a sign ambiguity of mass $\pm m$. Thus, in order to avoid this ambiguity, one

has to solve the first-order equation of $\epsilon_{\rho}^{\alpha \beta} \bar{\nabla}_{\alpha} \Phi_{\beta \mu \nu}+m \Phi_{\rho \mu \nu}=0$ itself under the transverse and traceless (TT) gauge condition.

It was known that the operator approach (method) [7, 8] is very useful to derive the quasinormal modes of spin-2 field of graviton in the BTZ black hole background in the framework of the cosmological TMG. This method has been applied to the new massive gravity to derive their quasinormal modes of the BTZ black hole [9]. Very recently, we have obtained quasinormal modes of the BTZ black hole in spin-3 TMG by using the operator method [10]. This method shows clearly how to derive quasinormal modes without any sign ambiguity in mass.

On the other hand, the presence of the logarithmic modes at the critical point of the TMG was pointed out [11, 12, 13]. In particular, Grumiller and Johansson [11] have shown that these modes grow linearly in time and the radial coordinate of the $\mathrm{AdS}_{3}$ spacetimes, which cause issues on the stability and the chiral nature of the theory. After their work, a derivation of the logarithmic quasinormal modes of spin-2 was performed for the BTZ back 
hole [14]. It seems that the operator approach is the only known method to derive the logarithmic quasinormal modes of spin-3 field because solving the second-order equation at the critical point cannot provide appropriate logarithmic quasinormal modes, in compared with the non-critical case.

In this work, we wish to derive logarithmic quasinormal modes and frequencies of a traceless spin-3 field around the BTZ black hole at the critical point of spin-3 topologically massive gravity theory. We will observe how they differ from the logarithmic quasinormal modes of a spin-2 field. Also, we explore Log-boundary conditions for left-and right-logarithmic modes. Finally, these quasinormal frequencies will be also confirmed by considering a logarithmic conformal field theory (LCFT).

\section{Perturbation analysis for spin-3 field}

\subsection{Action of spin-3 TMG}

The action for spin-3 coupled to TMG is given by

$$
\begin{gathered}
S=\frac{1}{8 \pi G} \int\left[e^{a} \wedge d \omega_{a}+\frac{1}{2} \epsilon_{a b c} e^{a} \wedge \omega^{b} \wedge \omega^{c}+\frac{1}{6 l^{2}} \epsilon_{a b c} e^{a} \wedge e^{b} \wedge e^{c}-2 \sigma e^{a b} \wedge d \omega_{a b}\right. \\
\left.-2 \sigma \epsilon_{a b c} e^{a} \wedge \omega^{b d} \wedge \omega_{d}^{c}-2 \sigma e^{a b} \wedge \epsilon_{(a \mid c d} \omega^{c} \wedge \omega_{\mid b)}{ }^{d}-\frac{2 \sigma}{l^{2}} \epsilon_{a b c} e^{a} \wedge e^{b d} \wedge e_{d}^{c}\right] \\
-\frac{1}{16 \pi G \mu} \int\left[\omega^{a} \wedge d \omega_{a}+\frac{1}{3} \epsilon_{a b c} \omega^{a} \wedge \omega^{b} \wedge \omega^{c}-2 \sigma \omega^{a b} \wedge d \omega_{a b}-4 \sigma \epsilon_{a b c} \omega^{a} \wedge \omega^{b d} \wedge \omega_{d}^{c}\right. \\
\left.+\beta^{a} \wedge T_{a}-2 \sigma \beta^{a b} \wedge T_{a b}\right],
\end{gathered}
$$

where $\sigma<0$ is a free parameter from $\mathrm{SL}(3, \mathrm{R})$ gauge group. Here two Lagrange multipliers

$$
\beta^{a}=\tilde{\beta}^{a}+\frac{e^{a}}{l^{2}}, \quad \beta^{a b}=\tilde{\beta}^{a b}+\frac{e^{a b}}{l^{2}}
$$

are introduced to impose the torsion free conditions [2]

$$
\begin{aligned}
T^{a} & \equiv d e^{a}+\epsilon^{a b c} \omega_{b} \wedge e_{c}-4 \sigma \epsilon^{a b c} e_{b d} \wedge \omega_{c}{ }^{d}=0, \\
T^{a b} & \equiv d e^{a b}+\epsilon^{c d(a \mid} \omega_{c} \wedge e_{d}^{\mid b)}+\epsilon^{c d(a \mid} e_{c} \wedge \omega_{d}^{\mid b)}=0 .
\end{aligned}
$$

The former in Eq. (1) denotes the action for the spin-3 $\mathrm{AdS}_{3}$ gravity [2], while the latter represents the spin-3 generalization of gravitational Chern-Simons term with a coupling constant $1 / \mu$. The equations of motion obtained by varying this action are given by the torsion free conditions

$$
T^{a}=0, \quad T^{a b}=0,
$$


and four equations

$$
\begin{aligned}
& R_{a}-\frac{1}{2 \mu}\left(d \beta_{a}+\epsilon_{a b c} \beta^{b} \wedge \omega^{c}-2 \sigma \epsilon_{(c \mid d a} \beta^{b c} \wedge \omega_{\mid b)}^{d}\right)=0 \\
& R_{a}+\frac{1}{2} \epsilon_{a b c}\left[\beta^{b} \wedge e^{c}-\frac{e^{b} \wedge e^{c}}{l^{2}}+4 \sigma\left(\frac{e^{b d} \wedge e_{d}^{c}}{l^{2}}-e^{b d} \wedge \beta_{d}^{c}\right)\right]=0, \\
& R_{a b}-\frac{1}{2 \mu}\left(d \beta_{a b}+\epsilon_{c d(a \mid} \beta^{c} \wedge \omega_{\mid b)}^{d}+\epsilon_{c d(a \mid} \omega^{c} \wedge \beta_{\mid b)}^{d}\right)=0 \\
& R_{a b}+\frac{1}{2}\left(\epsilon_{c d(a \mid} \beta^{c} \wedge e_{\mid b)}^{d}+\epsilon_{c d(a \mid} e^{c} \wedge \beta_{\mid b)}^{d}\right)-\frac{1}{l^{2}} \epsilon_{c d(a \mid} e^{c} \wedge e_{\mid b)}^{d}=0
\end{aligned}
$$

with

$$
\begin{aligned}
R_{a} & =d \omega_{a}+\frac{1}{2} \epsilon_{a b c}\left(\omega^{b} \wedge \omega^{c}+\frac{e^{b} \wedge e^{c}}{l^{2}}\right)-2 \sigma \epsilon_{a b c}\left(\omega_{b d} \wedge \omega_{c}^{d}+\frac{e_{b d} \wedge e_{c}^{d}}{l^{2}}\right) \\
R_{a b} & =d \omega_{a b}+\epsilon_{c d(a \mid} \omega^{c} \wedge \omega_{\mid b)}^{d}+\frac{1}{l^{2}} \epsilon_{c d(a \mid} e^{c} \wedge e_{\mid b)}^{d} .
\end{aligned}
$$

At this stage, we note that these differ from the pure gravity coupled to spin-3 field theory by $\beta^{a}$ and $\beta^{a b}$ terms. However, for

$$
\beta^{a}=\frac{e^{a}}{l^{2}}, \quad \beta^{a b}=\frac{e^{a b}}{l^{2}}
$$

the extra terms disappear due to the torsion free conditions, leading to the pure gravity coupled to spin-3 field theory [2]. This implies that the nonrotating BTZ black hole solution to pure gravity coupled to spin-3 field theory [15]

$$
\bar{e}^{a}=e_{\mathrm{BTZ}}^{a}
$$

with

$$
e_{\mathrm{BTZ}}^{0}=\left(-M+\frac{r^{2}}{l^{2}}\right) d t, \quad e_{\mathrm{BTZ}}^{1}=\left(-M+\frac{r^{2}}{l^{2}}\right)^{-1} d r, \quad e_{\mathrm{BTZ}}^{2}=r d \phi
$$

is also the solution to the above equations of motion. Here, the spin connection $\bar{\omega}^{a}=\bar{\omega}_{\mathrm{BTZ}}^{a}$ takes its components as

$$
\bar{\omega}_{\mathrm{BTZ}}^{0}=\frac{1}{r}\left(-M+\frac{r^{2}}{l^{2}}\right) \bar{e}^{2}, \quad \bar{\omega}_{\mathrm{BTZ}}^{1}=0, \quad \bar{\omega}_{\mathrm{BTZ}}^{2}=\frac{\partial}{\partial r}\left(-M+\frac{r^{2}}{l^{2}}\right) \bar{e}^{0} .
$$

In addition, one has

$$
\bar{\beta}^{a}=\frac{e_{\mathrm{BTZ}}^{a}}{l^{2}}, \quad \bar{e}^{a b}=e_{\mathrm{BTZ}}^{a b}=0, \quad \bar{\omega}^{a b}=\omega_{\mathrm{BTZ}}^{a b}=0, \quad \bar{\beta}^{a b}=\beta_{\mathrm{BTZ}}^{a b}=\frac{e_{\mathrm{BTZ}}^{a b}}{l^{2}}=0
$$

for the BTZ black hole. 


\subsection{Perturbation for spin-3 field}

Now we consider the perturbations around the BTZ black hole background with background fields $\bar{e}^{a}, \bar{\omega}^{a}, \bar{\beta}^{a}, \bar{e}^{a b}, \bar{\omega}^{a b}$, and $\bar{\beta}^{a b}$. For simplicity, we denote the perturbed fields as $e^{a}, \cdots$ without the bar notation $\left(^{-}\right)$. The six perturbed equations take the forms

$$
\begin{aligned}
d e^{a}+\epsilon^{a b c} \bar{\omega}_{b} \wedge e_{c}+\epsilon^{a b c} \omega_{b} \wedge \bar{e}_{c}=0 & =0 \\
d \omega_{a}+\epsilon_{a b c}\left(\bar{\omega}^{b} \wedge \omega^{c}+\frac{\bar{e}^{b} \wedge e^{c}}{l^{2}}\right)-\frac{1}{2 \mu}\left(d \beta_{a}+\epsilon_{a b c} \bar{\beta}^{b} \wedge \omega^{c}+\epsilon_{a b c} \beta^{b} \wedge \bar{\omega}^{c}\right) & =0 \\
d \omega_{a}+\epsilon_{a b c}\left(\bar{\omega}^{b} \wedge \omega^{c}+\frac{\bar{e}^{b} \wedge e^{c}}{l^{2}}\right)+\frac{1}{2} \epsilon_{a b c}\left[\bar{\beta}^{b} \wedge e^{c}+\beta^{b} \wedge \bar{e}^{c}-\frac{2}{l^{2}} \bar{e}^{b} \wedge e^{c}\right] & =0 \\
d e^{a b}+\epsilon^{c d(a \mid} \bar{\omega}_{c} \wedge e_{d}^{\mid b)}+\epsilon^{c d(a \mid} \bar{e}_{c} \wedge \omega_{d}^{\mid b)} & =0 \\
R_{a b}-\frac{1}{2 \mu}\left(d \beta_{a b}+\epsilon_{c d(a \mid} \bar{\beta}^{c} \wedge \omega_{\mid b)}^{d}+\epsilon_{c d(a \mid} \bar{\omega}^{c} \wedge \beta_{\mid b)}^{d}\right) & =0 \\
R_{a b}+\frac{1}{2}\left(\epsilon_{c d(a \mid} \bar{\beta}^{c} \wedge e_{\mid b)}^{d}+\epsilon_{c d(a \mid} \bar{e}^{c} \wedge \beta_{\mid b)}^{d}\right)-\frac{1}{l^{2}} \epsilon_{c d(a \mid} \bar{e}^{c} \wedge e_{\mid b)}^{d} & =0
\end{aligned}
$$

with the perturbed Ricci tensor

$$
R_{a b}=d \omega_{a b}+\epsilon_{c d(a \mid} \bar{\omega}^{c} \wedge \omega_{\mid b)}^{d}+\frac{1}{l^{2}} \epsilon_{c d(a \mid} \bar{e}^{c} \wedge e_{\mid b)}^{d} .
$$

Hereafter, we will express the perturbed fields in terms of the frame fields $h_{\mu \nu}$ and $\Phi_{\mu \nu \lambda}$ as

$$
h_{\mu \nu}=e_{\mu a} \bar{e}_{\nu}^{a}, \quad \Phi_{\mu \nu \lambda}=e_{\mu a b} \bar{e}_{\nu}^{a} \bar{e}_{\lambda}^{b},
$$

where the Latin indices of $a$ and $b$ are replaced by the Greek indices of $\nu$ and $\lambda$. The Greek indices are raised (or lowered) by the BTZ metric of $\bar{g}_{\mu \nu}^{\mathrm{BTZ}}=\bar{e}_{\mu}^{a} \bar{e}_{\nu}^{b} \eta_{a b}$ where

$$
d s_{\mathrm{BTZ}}^{2}=\bar{g}_{\mu \nu}^{\mathrm{BTZ}} d x^{\mu} d x^{\nu}=-\left(-M+\frac{r^{2}}{l^{2}}\right) d t^{2}+\frac{d r^{2}}{\left(-M+\frac{r^{2}}{l^{2}}\right)}+r^{2} d \phi^{2} .
$$

The perturbed equation of spin-2 graviton takes the form [5]

$$
\left(\bar{\square}+\frac{2}{l^{2}}\right) h_{\sigma}^{\rho}+\frac{1}{\mu} \epsilon^{\rho \mu \nu} \bar{\nabla}_{\mu}\left(\bar{\square}+\frac{2}{l^{2}}\right) h_{\nu \sigma}=0,
$$

which is decoupled completely from the spin-3 perturbed equation as [3]

$$
\bar{\square} \Phi^{\rho \alpha \beta}+\frac{1}{2 \mu} \epsilon^{\rho \mu \nu} \bar{\nabla}_{\mu} \square \Phi_{\nu}^{\alpha \beta}=0 .
$$

In this work, we consider the BTZ black hole with the mass $M=1$ and the $\mathrm{AdS}_{3}$ curvature radius $l=1$ in global coordinates as

$$
d s_{\mathrm{BTZ}}^{2}=\bar{g}_{\mu \nu} d x^{\mu} d x^{\nu}=-\sinh ^{2} \rho d \tau^{2}+\cosh ^{2} \rho d \phi^{2}+d \rho^{2},
$$


where the event horizon is located at $\rho=0$, while the infinity is at $\rho=\infty$. Introducing the light-cone coordinates $u / v=\tau \pm \phi$, the metric tensor $\bar{g}_{\mu \nu}$ takes the form

$$
\bar{g}_{\mu \nu}=\left(\begin{array}{ccc}
\frac{1}{4} & -\frac{1}{4} \cosh 2 \rho & 0 \\
-\frac{1}{4} \cosh 2 \rho & \frac{1}{4} & 0 \\
0 & 0 & 1
\end{array}\right)
$$

Then the metric tensor (28) admits the Killing vector fields $L_{k}(k=0,-1,1)$ for the local $\mathrm{SL}(2, \mathrm{R}) \times \mathrm{SL}(2, \mathrm{R})$ algebra as

$$
L_{0}=-\partial_{u}, \quad L_{-1 / 1}=e^{\mp u}\left[-\frac{\cosh 2 \rho}{\sinh 2 \rho} \partial_{u}-\frac{1}{\sinh 2 \rho} \partial_{v} \mp \frac{1}{2} \partial_{\rho}\right],
$$

and $\bar{L}_{0}$ and $\bar{L}_{-1 / 1}$ are obtained by substituting $u \leftrightarrow v$. Locally, they form a basis of the $\mathrm{SL}(2, \mathrm{R})$ Lie algebra as

$$
\left[L_{0}, L_{ \pm 1}\right]=\mp L_{ \pm 1}, \quad\left[L_{1}, L_{-1}\right]=2 L_{0} .
$$

Here, we note that the totally symmetric spin-3 field $\Phi^{\rho \mu \nu}$ satisfying the TT gauge condition

$$
\bar{\nabla}^{\mu} \Phi_{\mu \nu \rho}=0, \quad \Phi_{\mu}^{\mu \nu}=0
$$

has only one degree of freedom corresponding to a single massive mode propagating in the BTZ black hole spacetimes [10].

On the other hand, the third-order equation (26) can also be expressed as

$$
\left(\mathcal{D}^{M} \mathcal{D}^{L} \mathcal{D}^{R} \Phi\right)^{\rho \mu \nu}=0
$$

in terms of mutually commuting operators

$$
\left(\mathcal{D}^{L / R}\right)^{\rho \nu}=\delta^{\rho \nu} \pm \frac{1}{2} \epsilon^{\rho \mu \nu} \bar{\nabla}_{\mu}, \quad\left(\mathcal{D}^{M}\right)^{\rho \nu}=\delta^{\rho \nu}+\frac{1}{2 \mu} \epsilon^{\rho \mu \nu} \bar{\nabla}_{\mu} .
$$

We note that Eq. (32) is reduced to Eq. (26) when using the BTZ background

$$
\bar{R}_{\rho \sigma \mu \nu}=-\left(\bar{g}_{\rho \mu} \bar{g}_{\sigma \nu}-\bar{g}_{\rho \nu} \bar{g}_{\sigma \mu}\right), \quad \bar{R}_{\mu \nu=}=-2 \bar{g}_{\mu \nu},
$$

together with the TT gauge condition and the relation of $\left[\bar{\nabla}_{\mu}, \bar{\nabla}_{\nu}\right] \Phi^{\mu \alpha \beta}=-4 \Phi_{\nu}^{\alpha \beta}$. Therefore, the third-order equation (26) can be decomposed into three first-order differential equations:

$$
\left(\mathcal{D}^{M} \Phi\right)^{\rho \mu \nu}=0, \quad\left(\mathcal{D}^{L} \Phi\right)^{\rho \mu \nu}=0, \quad\left(\mathcal{D}^{R} \Phi\right)^{\rho \mu \nu}=0,
$$

for a massive, a left-moving, and a right-moving degree of freedom, respectively. Importantly, three first-order differential equations (35) can be simply rewritten in terms of a single massive first-order differential equation as

$$
\epsilon_{\rho}^{\alpha \beta} \bar{\nabla}_{\alpha} \Phi_{\beta \mu \nu}+m \Phi_{\rho \mu \nu}=0
$$


with $m=2 \mu, 2$, and -2 .

It seems appropriate to comment that it could also be expressed in terms of a second-order differential equation [6] as

$$
\left[\square^{2}-m^{2}+4\right] \Phi_{\rho \mu \nu}=0 .
$$

However, there exists a sign ambiguity $\pm m$ in this equation. Therefore, in order to avoid this ambiguity, we will directly solve the first-order equation (36) with the TT gauge condition. Note that at the chiral (critical) point of $\mu=1$, the operators $\mathcal{D}^{M}$ and $\mathcal{D}^{L}$ degenerate.

Having the structure in mind, let us find quasinormal modes for the spin-3 field in the BTZ background by solving (36) together with the TT gauge condition. In order to implement the operator method [7, 9], one has to choose either the anti-chiral highest weight condition of $L_{1} \Phi_{\rho \mu \nu}=0$ or the chiral highest weight condition of $\bar{L}_{1} \Phi_{\rho \mu \nu}=0$, but not both simultaneously. Actually, we note that for a generic symmetric tensor $\Phi_{\rho \mu \nu}$, the transversality condition of $\bar{\nabla}^{\mu} \Phi_{\mu \nu \rho}=0$ is not equivalent to choosing the chiral (anti-chiral) highest weight condition. However, selecting proper components of $\Phi_{\rho \mu \nu}$, two are equivalent to each other.

\section{Left-logarithmic quasinormal modes}

We observe that similar to the perturbed equation (25) for the spin-2 graviton, the spin3 fluctuation also satisfies a third-order differential equation (26). Since the logarithmic quasinormal modes of the graviton were computed at the critical point in [14, we wish to calculate logarithmic quasinormal modes of the spin-3 field by the operator method in this section.

\subsection{Logarithmic quasinormal modes}

By solving the first-order equation (36), we obtain the left-moving solution of a anti-chiral highest weight field

$$
\Phi_{\rho \mu \nu}^{L}(u, v, \rho)=e^{i k(t-\phi)-2 h_{L}(m) t}(\sinh \rho)^{-2 h_{L}(m)}(\tanh \rho)^{i k} F_{\rho \mu \nu}^{L}(\rho),
$$


where $h_{L}(m)=(m-2) / 2$ and $F_{\rho \mu \nu}^{L}(\rho)$ is given by [10]

$$
\begin{aligned}
& F_{u \mu \nu}^{L}(\rho)=\left(\begin{array}{ccc}
0 & 0 & 0 \\
0 & 0 & 0 \\
0 & 0 & 0
\end{array}\right)_{\mu \nu}, \\
& F_{v \mu \nu}^{L}(\rho)=\left(\begin{array}{ccc}
0 & 0 & 0 \\
0 & 1 & \frac{2}{\sinh 2 \rho} \\
0 & \frac{2}{\sinh 2 \rho} & \frac{4}{\sinh ^{2} 2 \rho}
\end{array}\right)_{\mu \nu}, \\
& F_{\rho \mu \nu}^{L}(\rho)=\left(\begin{array}{ccc}
0 & 0 & 0 \\
0 & \frac{2}{\sinh ^{2} \rho} & \frac{4}{\sinh ^{2} 2 \rho} \\
0 & \frac{4}{\sinh ^{2} 2 \rho} & \frac{8}{\sinh ^{3} 2 \rho}
\end{array}\right)_{\mu \nu} .
\end{aligned}
$$

Note here that $F_{v \mu \nu}^{L}(\rho)$ takes the same form as the spin-2 graviton which is Eq. (17) in Ref. [8], while $F_{u \mu \nu}^{L}(\rho)$ is null and $F_{\rho \mu \nu}^{L}(\rho)$ is more damped than $F_{v \mu \nu}^{L}(\rho)$ for large $\rho$.

However, the basis of solutions (38) becomes inadequate at the chiral point $\mu=1$, since the $L$ and $M$ branches coincide at this point from Eq. (33). One could remedy this problem by constructing a new mode solution satisfying [1]

$$
\mathcal{D}^{L} \Phi_{\rho \mu \nu}^{L, n e w}=-\Phi_{\rho \mu \nu}^{L} \neq 0
$$

so that

$$
\mathcal{D}^{L} \mathcal{D}^{L} \Phi_{\rho \mu \nu}^{L, \text { new }}=-\mathcal{D}^{L} \Phi_{\rho \mu \nu}^{L}=0,
$$

while satisfying with the anti-chiral highest weight condition as well as the TT gauge condition.

Then, at the critical point of $\mu=1(m=2)$, a new logarithmic solution is given by

$$
\begin{aligned}
\Phi_{\rho \mu \nu}^{L, \text { new }} & =\left.\partial_{m} \Phi_{\rho \mu \nu}^{L, m}\right|_{m \rightarrow 2} \\
& =\left.y(t, \rho) \Phi_{\rho \mu \nu}^{L}(u, v, \rho)\right|_{m \rightarrow 2},
\end{aligned}
$$

where

$$
y(t, \rho)=-t-\ln \sinh \rho .
$$

Here, $\left.\Phi_{\rho \mu \nu}^{L}(u, v, \rho)\right|_{m \rightarrow 2}$ is given by Eq. (38) as

$$
\left.\Phi_{\rho \mu \nu}^{L}(u, v, \rho)\right|_{m \rightarrow 2}=e^{i k(t-\phi)}(\tanh \rho)^{i k} F_{\rho \mu \nu}^{L}(\rho) .
$$

Next, according to Sachs's proposal [14], the logarithmic quasinormal modes can be constructed by using the following operation

$$
\Phi_{\rho \mu \nu}^{L(n), n e w}(u, v, \rho)=\left(\bar{L}_{-1} L_{-1}\right)^{n} \Phi_{\rho \mu \nu}^{L, n e w}(u, v, \rho),
$$


which means that we should compute their descendants of $\Phi_{\rho \mu \nu}^{L, n e w}(u, v, \rho)$ by using the operator method.

The first descendants of $\Phi_{\rho \mu \nu}^{L, n e w}(u, v, \rho)$ represented by

$$
\Phi_{\rho \mu \nu}^{L(1), n e w}(u, v, \rho)=\left(\bar{L}_{-1} L_{-1}\right) \Phi_{\rho \mu \nu}^{L, n e w}(u, v, \rho)
$$

are explicitly given by

$$
\begin{aligned}
& \left(\bar{L}_{-1} L_{-1}\right) \Phi_{u \mu \nu}^{L, n e w}(u, v, \rho)=\frac{e^{-2 t}}{\sinh ^{2} \rho} e^{i k v}(\tanh \rho)^{i k}\left(\begin{array}{ccc}
0 & 0 & 0 \\
0 & f_{u v v}^{L(1)} & \frac{2 f_{u v v}^{L(1)}}{\sinh 2 \rho} \\
0 & \frac{2 f_{u v v}^{L(1)}}{\sinh 2 \rho} & \frac{4 f_{u v v}^{L(1)}}{\sinh ^{2} 2 \rho}
\end{array}\right)_{\mu \nu}, \\
& \left(\bar{L}_{-1} L_{-1}\right) \Phi_{v \mu \nu}^{L, n e w}(u, v, \rho)=\frac{e^{-2 t}}{\sinh ^{2} \rho} e^{i k v}(\tanh \rho)^{i k}\left(\begin{array}{ccc}
0 & f_{u v v}^{L(1)} & \frac{2 f_{u v v}^{L(1)}}{\sinh 2 \rho} \\
f_{u v v}^{L(1)} & \frac{f_{v v v}^{L(1)}}{2} & \frac{f_{v v \rho}^{L(1)}}{\sinh 2 \rho} \\
\frac{2 f_{u v v}^{L(1)}}{\sinh 2 \rho} & \frac{f_{v v \rho}^{L(1)}}{\sinh 2 \rho} & \frac{2 f_{v \rho \rho}^{L(1)}}{\sinh ^{2} 2 \rho}
\end{array}\right)_{\mu \nu} \\
& \left(\bar{L}_{-1} L_{-1}\right) \Phi_{\rho \mu \nu}^{L, n e w}(u, v, \rho)=\frac{e^{-2 t}}{\sinh ^{2} \rho} e^{i k v}(\tanh \rho)^{i k}\left(\begin{array}{ccc}
0 & \frac{2 f_{u v v}^{L(1)}}{\sinh 2 \rho} & \frac{4 f_{u v v}^{L(1)}}{\sinh ^{2} 2 \rho} \\
\frac{2 f_{u v v}^{L(1)}}{\sinh 2 \rho} & \frac{f_{v v \rho}^{L(1)}}{\sinh 2 \rho} & \frac{2 f_{v \rho}^{L(1)}}{\sinh ^{2} 2 \rho} \\
\frac{4 f_{u v v}^{L(1)}}{\sinh ^{2} 2 \rho} & \frac{2 f_{v \rho \rho}^{L(1)}}{\sinh ^{2} 2 \rho} & \frac{4 f_{\rho \rho}^{L(1)}}{\sinh ^{3} 2 \rho}
\end{array}\right)_{\mu \nu}
\end{aligned}
$$

whose relevant components are given by

$$
\begin{aligned}
& f_{u v v}^{L(1)}=1+(3-i k) y(t, \rho) \\
& f_{v v v}^{L(1)}=5-3 i k+(3-i k) \cosh 2 \rho+2\left(3-4 i k-k^{2}\right) y(t, \rho) \\
& f_{v v \rho}^{L(1)}=5-3 i k+(5-i k) \cosh 2 \rho+2\left(3-4 i k-k^{2}+(3-i k) \cosh 2 \rho\right) y(t, \rho), \\
& f_{v \rho \rho}^{L(1)}=5-3 i k+(7-i k) \cosh 2 \rho+2\left(3-4 i k-k^{2}+2(3-i k) \cosh 2 \rho\right) y(t, \rho), \\
& f_{\rho \rho \rho}^{L(1)}=5-3 i k+(9-i k) \cosh 2 \rho+2\left(3-4 i k-k^{2}+3(3-i k) \cosh 2 \rho\right) y(t, \rho) .
\end{aligned}
$$

Then, the second descendants of $\Phi_{\rho \mu \nu}^{L, n e w}(u, v, \rho)$ are read off from the operation

$$
\Phi_{\rho \mu \nu}^{L(2), n e w}(u, v, \rho)=\left(\bar{L}_{-1} L_{-1}\right)^{2} \Phi_{\rho \mu \nu}^{L, n e w}(u, v, \rho) .
$$


We have their explicit forms

$$
\begin{aligned}
& \left(\bar{L}_{-1} L_{-1}\right)^{2} \Phi_{u \mu \nu}^{L, \text { new }}(u, v, \rho)=\frac{e^{-4 t}}{\sinh ^{4} \rho} e^{i k v}(\tanh \rho)^{i k}\left(\begin{array}{ccc}
0 & f_{\text {uuv }}^{L(2)} & \frac{f_{u u \rho}^{L(2)}}{\sinh 2 \rho} \\
f_{u u v}^{L(2)} & f_{u v v}^{L(2)} & \frac{f_{u v \rho}^{L(2)}}{\sinh 2 \rho} \\
\frac{f_{u u \rho}^{L(2)}}{\sinh 2 \rho} & \frac{f_{u v \rho}^{L(2)}}{\sinh 2 \rho} & \frac{f_{u \rho \rho}^{L(2)}}{\sinh ^{2} 2 \rho}
\end{array}\right)_{\mu \nu} \\
& \left(\bar{L}_{-1} L_{-1}\right)^{2} \Phi_{v \mu \nu}^{L, \text { new }}(u, v, \rho)=\frac{e^{-4 t}}{\sinh ^{4} \rho} e^{i k v}(\tanh \rho)^{i k}\left(\begin{array}{ccc}
f_{u u v}^{L(2)} & f_{u v v}^{L(2)} & \frac{f_{u v \rho}^{L(2)}}{\sinh 2 \rho} \\
f_{u v v}^{L(2)} & f_{v v v}^{L(2)} & \frac{f_{v v \rho}^{L(2)}}{\sinh 2 \rho} \\
\frac{f_{u v \rho}^{L(2)}}{\sinh 2 \rho} & \frac{f_{v v \rho}^{L(2)}}{\sinh 2 \rho} & \frac{f_{v \rho \rho}^{L(2)}}{\sinh ^{2} 2 \rho}
\end{array}\right) \text {, } \\
& \left(\bar{L}_{-1} L_{-1}\right)^{2} \Phi_{\rho \mu \nu}^{L, \text { new }}(u, v, \rho)=\frac{e^{-4 t}}{\sinh ^{4} \rho} e^{i k v}(\tanh \rho)^{i k}\left(\begin{array}{ccc}
\frac{f_{u u \rho}^{L(2)}}{\sinh 2 \rho} & \frac{f_{u v \rho}^{L(2)}}{\sinh 2 \rho} & \frac{f_{u \rho \rho}^{L(2)}}{\sinh ^{2} 2 \rho} \\
\frac{f_{u v \rho}^{L(2)}}{\sinh 2 \rho} & \frac{f_{v v \rho}^{L(2)}}{\sinh 2 \rho} & \frac{f_{v \rho \rho}^{L(2)}}{\sinh ^{2} 2 \rho} \\
\frac{f_{u \rho \rho}^{L(2)}}{\sinh ^{2} 2 \rho} & \frac{f_{v \rho \rho}^{L(2)}}{\sinh ^{2} 2 \rho} & \frac{f_{\rho \rho \rho}^{L(2)}}{\sinh ^{3} 2 \rho}
\end{array}\right)_{\mu \nu}
\end{aligned}
$$

The full expressions of the matrix elements, $f_{\text {uuv }}^{L(2)}$, etc., are listed in Appendix A1.

On the the hand, the third descendants of $\Phi_{\rho \mu \nu}^{L, n e w}(u, v, \rho)$ are given by

$$
\Phi_{\rho \mu \nu}^{L(3), n e w}(u, v, \rho)=\left(\bar{L}_{-1} L_{-1}\right)^{3} \Phi_{\rho \mu \nu}^{L, n e w}(u, v, \rho) .
$$

We have

$$
\begin{aligned}
& \left(\bar{L}_{-1} L_{-1}\right)^{3} \Phi_{u \mu \nu}^{L, n e w}(u, v, \rho)=\frac{e^{-6 t}}{\sinh ^{6} \rho} e^{i k v}(\tanh \rho)^{i k}\left(\begin{array}{ccc}
f_{u u u}^{L(3)} & f_{u u v}^{L(3)} & \frac{f_{u u \rho}^{L(2)}}{\sinh 2 \rho} \\
f_{u u v}^{L(3)} & f_{u v v}^{L(3)} & \frac{f_{u v \rho}^{L(3)}}{\sinh 2 \rho} \\
\frac{f_{u u \rho}^{L(2)}}{\sinh 2 \rho} & \frac{f_{u v \rho}^{L(3)}}{\sinh 2 \rho} & \frac{f_{u \rho \rho}^{L(3)}}{\sinh ^{2} 2 \rho}
\end{array}\right)_{\mu \nu} \\
& \left(\bar{L}_{-1} L_{-1}\right)^{3} \Phi_{v \mu \nu}^{L, \text { new }}(u, v, \rho)=\frac{e^{-6 t}}{\sinh ^{6} \rho} e^{i k v}(\tanh \rho)^{i k}\left(\begin{array}{ccc}
f_{u u v}^{L(3)} & f_{u v v}^{L(3)} & \frac{f_{u v \rho}^{L(3)}}{\sinh 2 \rho} \\
f_{u v v}^{L(3)} & f_{v v v}^{L(3)} & \frac{f_{v v \rho}^{L(3)}}{\sinh 2 \rho} \\
\frac{f_{u v \rho}^{L(3)}}{\sinh 2 \rho} & \frac{f_{v v \rho}^{L(3)}}{\sinh 2 \rho} & \frac{f_{v \rho \rho}^{L(3)}}{\sinh ^{2} 2 \rho}
\end{array}\right)_{\mu \nu} \\
& \left(\bar{L}_{-1} L_{-1}\right)^{3} \Phi_{\rho \mu \nu}^{L, \text { new }}(u, v, \rho)=\frac{e^{-6 t}}{\sinh ^{6} \rho} e^{i k v}(\tanh \rho)^{i k}\left(\begin{array}{ccc}
\frac{f_{u u \rho}^{L(3)}}{\sinh 2 \rho} & \frac{f_{u v \rho}^{L(3)}}{\sinh 2 \rho} & \frac{f_{u \rho \rho}^{L(3)}}{\sinh ^{2} 2 \rho} \\
\frac{f_{u v \rho}^{L(3)}}{\sinh 2 \rho} & \frac{f_{v v \rho}^{L(3)}}{\sinh 2 \rho} & \frac{f_{v \rho \rho}^{L(3)}}{\sinh ^{2} 2 \rho} \\
\frac{f_{u \rho \rho}^{L(3)}}{\sinh ^{2} 2 \rho} & \frac{f_{v \rho \rho}^{L(3)}}{\sinh ^{2} 2 \rho} & \frac{f_{\rho \rho \rho}^{L(3)}}{\sinh ^{3} 2 \rho}
\end{array}\right)_{\mu \nu}
\end{aligned}
$$

Here again, the full expressions of $f_{u u u}^{L(3)}, \cdots$, are written down in Appendix A2. The fourth descendants are given in Appendix A3 with $s$-mode $(k=0)$.

From these expressions, one can deduce the expression for higher order of the descendants 
as

$$
\begin{aligned}
\Phi_{\rho \mu \nu}^{L(n), \text { new }}(u, v, \rho) & =\left(\bar{L}_{-1} L_{-1}\right)^{n} \Phi_{\rho \mu \nu}^{L, \text { new }}(u, v, \rho) \\
& =\frac{e^{-2 n t}}{\sinh ^{2 n} \rho} e^{i k v}(\tanh \rho)^{i k} F_{\rho \mu \nu}^{L(n)}(\rho)
\end{aligned}
$$

where $F_{\rho \mu \nu}^{L(n)}(\rho)$ is the corresponding $n$-th order matrix. As a result, we read off the leftlogarithmic quasinormal frequencies of a traceless spin-3 field from the quasinormal modes (59)

$$
\omega_{L}^{n}=-k-2 i n, \quad n \in N
$$

which is the same expression for spin-2 graviton $h_{\mu \nu}$ [14]. This is one of our main results.

It is by now appropriate to comment on the right-moving solution and the right-logarithmic quasinormal modes. The right-moving solution and its corresponding logarithmic solution can be easily constructed by the substitution of both $u \leftrightarrow v, L \leftrightarrow R$ and $\phi \rightarrow-\phi, k \rightarrow-k$ in Eqs. (38), (39), and (44). Moreover, the succeeding descendants of the right-logarithmic quasinormal modes can also be derived by the mentioned substitution, and finally yield the quasinormal frequencies as

$$
\omega_{R}^{n}=k-2 i n, \quad n \in N
$$

\subsection{Log-boundary conditions}

Since the time dependent part of the solution (59) is simply given by exponential fall-off in $t$ as $\left[e^{-2 n t}\right]$ whereas the radial part is a complicated form for each descendant, it would be better to observe their asymptotic behaviors. For this purpose, let us find the asymptotic behaviors of the left-logarithmic solutions (44). We have the asymptotic form in the $\rho \rightarrow \infty$ limit as

$$
\begin{aligned}
\Phi_{u \mu \nu}^{L(0), \text { new }, \infty}(u, v, \rho) & \sim\left(\begin{array}{lll}
0 & 0 & 0 \\
0 & 0 & 0 \\
0 & 0 & 0
\end{array}\right)_{\mu \nu}, \\
\Phi_{v \mu \nu}^{L(0), \text { new }, \infty}(u, v, \rho) & \sim-\rho\left(\begin{array}{ccc}
0 & 0 & 0 \\
0 & 1 & e^{-2 \rho} \\
0 & e^{-2 \rho} & e^{-4 \rho}
\end{array}\right)_{\mu \nu}, \\
\Phi_{\rho \mu \nu}^{L(0), \text { new }, \infty}(u, v, \rho) & \sim-\rho\left(\begin{array}{ccc}
0 & 0 & 0 \\
0 & e^{-2 \rho} & e^{-4 \rho} \\
0 & e^{-4 \rho} & e^{-6 \rho}
\end{array}\right)_{\mu \nu} .
\end{aligned}
$$


The second component $\Phi_{v \mu \nu}^{L(0), n e w, \infty}$ in Eq. (62) takes the same form as that of the spin-2 graviton [14. We point out that since the mode of $\Phi_{v v v}^{L(0), n e w, \infty}(\propto \rho)$ is growing in $\rho$, it could not be considered as a quasinormal mode. It may be cured by taking descendants. For example, taking the third descendants, we have

$$
\begin{gathered}
\Phi_{u \mu \nu}^{L(3), n e w, \infty}(u, v, \rho) \sim-\rho\left(\begin{array}{lll}
e^{-6 \rho} & e^{-4 \rho} & e^{-6 \rho} \\
e^{-4 \rho} & e^{-2 \rho} & e^{-4 \rho} \\
e^{-6 \rho} & e^{-4 \rho} & e^{-6 \rho}
\end{array}\right)_{\mu \nu}, \\
\Phi_{v \mu \nu}^{L(3), n e w, \infty}(u, v, \rho) \sim-\rho\left(\begin{array}{lll}
e^{-4 \rho} & e^{-2 \rho} & e^{-4 \rho} \\
e^{-2 \rho} & -\frac{1}{\rho} & e^{-2 \rho} \\
e^{-4 \rho} & e^{-2 \rho} & e^{-4 \rho}
\end{array}\right)_{\mu \nu}, \\
\Phi_{\rho \mu \nu}^{L(3), n e w, \infty}(u, v, \rho) \sim-\rho\left(\begin{array}{lll}
e^{-6 \rho} & e^{-4 \rho} & e^{-6 \rho} \\
e^{-4 \rho} & e^{-2 \rho} & e^{-4 \rho} \\
e^{-6 \rho} & e^{-4 \rho} & e^{-6 \rho}
\end{array}\right)_{\mu \nu} .
\end{gathered}
$$

We also would like to mention that all higher order components $\left\{\Phi_{v v v}^{L(n), n e w, \infty} \sim 1\right\}$ with $n>0$ are not dominant at large $\rho$, which implies that it may induce difficulty in identifying the corresponding dual operator on the LCFT side. On the other hand, all other components $\left\{\Phi_{\rho \mu \nu}^{L(n), n e w, \infty}\right\}$ with $n \geq 0$ for $\rho, \mu, \nu \neq v$ show the exponential fall-off in $\rho$ as $\left[\rho \cdot e^{-2 c \rho}\right]$ with $c=2,4,6$, which indicates genuine gravitational quasinormal modes.

Finally, it seems appropriate to comment on the fourth descendants $\Phi_{\lambda \mu \nu}^{L(4), n e w, \infty}(u, v, \rho)$. Their asymptotic behavior is exactly the same with the asymptotic form (63) of the third descendants. Thus, we expect that all higher order descendants with $n>4$ for the spin-3 case behave as like the third descendants have.

We have also proven that as were shown in Appendix B, these properties persist to the noncritical cases of $\mu \neq \pm 1$.

\section{AdS/LCFT correspondence}

The log gravity at the chiral point could be dual to a LCFT on the boundary described by $(\tau, \sigma)[11,13]$. In this section, we show how to derive quasinormal frequencies $\omega_{L / R}^{n}=\mp k-2 i n$ of the spin-3 field from the $\mathrm{LCFT}_{L}$ on the boundary. It was known that the spin-3 chiral gravity with the Brown-Henneaux boundary condition [16] is holographically dual to the $\mathrm{CFT}_{L}$ with classical $W_{3}$ algebra and central charge $c_{L}=3 l / G$ [4]. However, this is not our case because we did not require the Brown-Henneaux boundary condition. 
The $\mathrm{LCFT}_{L}$ [17, 18, 19] may arise from the two operators $C$ and $D$ which satisfy the degenerate eigenequations of $L_{0}$ as

$$
L_{0}\left|C>=h_{L}\right| C>, \quad L_{0}\left|D>=h_{L}\right| D>+\mid C>.
$$

The two-point functions of these operators take the forms

$$
<C(x) C(0)>=0, \quad<C(x) D(0)>=\frac{c}{x^{2 h_{L}}}, \quad<D(x) D(0)>=\frac{1}{x^{2 h_{L}}}[d-2 c \log (x)] .
$$

We note that Eq. (65) does not fix $C$ and its logarithmic partner $D$ uniquely. For example, $D^{\prime}=D+a C$ also satisfies Eq. (64). This freedom could be used to adjust the constant $d$ to any suitable value.

In order to derive quasinormal modes, we focus at the location of of the poles in the momentum space for the retarded two-point functions $G_{R}^{C C}(\tau, \sigma), G_{R}^{C D}(\tau, \sigma)$ and $G_{R}^{D D}(\tau, \sigma)$ [14]. It is very important to recognize that $G_{R}^{C D}(\tau, \sigma)$ is identical with that of the two point function in the CFT [20]. The momentum space representation can be read off from the commutator whose pole structure is given by

$$
\mathcal{D}^{D C}\left(p_{+}\right) \propto \Gamma\left(h_{L}+i \frac{p_{+}}{2 \pi T_{L}}\right) \Gamma\left(h_{L}-i \frac{p_{+}}{2 \pi T_{L}}\right),
$$

where $h_{L}=(m-2) / 2, p_{+}=(\omega+k) / 2$, and $T_{L}=r_{+} / 2 \pi=l \sqrt{M} / 2 \pi=1 / 2 \pi$ for the nonrotating BTZ black hole with $M=1$ and $l=1$. This function has poles in both the upper and lower half of the $\omega$-plane. It turned out that the poles located in the lower halfplane are the same as the poles of the retarded two-point function $G_{R}^{C D}(\tau, \sigma)$. Restricting the poles in Eq. (66) to the lower half-plane, we find one set of simple poles

$$
\omega_{L}=-k-2 i\left(n+h_{L}\right)
$$

with $n \in N$. This set of poles characterizes the decay of the perturbation on the $\mathrm{LCFT}_{L}$ side. Furthermore, $G_{R}^{D D}(t, \sigma)$ can be inferred by noting [18, 19]

$$
<D(x) D(0)>=\frac{\partial}{\partial h_{L}}<C(x) D(0)>.
$$

Then, this implies that its momentum space representation takes the form

$$
\mathcal{D}^{D D}\left(p_{+}\right) \propto \Gamma^{\prime}\left(h_{L}+i p_{+}\right) \Gamma\left(h_{L}-i p_{+}\right)+\Gamma\left(h_{L}+i p_{+}\right) \Gamma^{\prime}\left(h_{L}-i p_{+}\right),
$$

where the prime $\left(^{\prime}\right)$ denotes the differentiation with respect to $h_{L}$. The poles in the lower half plane are relevant to deriving quasinormal modes. We mention that $\mathcal{D}_{R}^{D D}\left(p_{+}\right)$has double poles, while $\mathcal{D}_{R}^{C D}\left(p_{+}\right)$has simple poles at the same location. These double poles are 
responsible for the linear-time dependence in $y(t, \rho)[=-t-\ln \sinh \rho]$ of the corresponding quasinormal modes.

Now, we are in a position to assign the bulk perturbation to $C$ and $D$. For $m>2$, the spin-3 perturbation $\Phi_{\rho \mu \nu}^{L}(m)$ is dual to a non-degenerate boundary operator $C$ with conformal weight $h_{L}=\frac{m-2}{2}$. At the chiral point of $m=2$, the perturbation of $\left.\Phi_{\rho \mu \nu}^{L}(m)\right|_{m \rightarrow 2}$ becomes a pure gauge. In this case, Eq. (64) together with the relation of $L_{1} D=0$ implies for the corresponding bulk perturbation [19]

$$
\Phi_{\rho \mu \nu}^{L, \text { new }}=\left.[y(t, \rho)+a] \Phi_{\rho \mu \nu}^{L}(m)\right|_{m \rightarrow 2},
$$

which shows that $\Phi_{\rho \mu \nu}^{L, \text { new }}$ is the bulk perturbation for the logarithmic partner $D$. The bulkboundary correspondence is summarized as

$$
\left.\Phi_{\rho \mu \nu}^{L}(m)\right|_{m \rightarrow 2} \longleftrightarrow C, \quad \Phi_{\rho \mu \nu}^{L, n e w} \longleftrightarrow D
$$

Similarly, we have the bulk-boundary correspondence for the right-movers as

$$
\left.\Phi_{\rho \mu \nu}^{R}(m)\right|_{m \rightarrow 2} \longleftrightarrow \tilde{C}, \quad \Phi_{\rho \mu \nu}^{R, n e w} \longleftrightarrow \tilde{D}
$$

if one introduces the right sector of $\mathrm{LCFT}_{R}$ operator $\tilde{C}$ and $\tilde{D}$ which satisfy the degenerate eigenequations for $\bar{L}_{0}$ as

$$
\bar{L}_{0}\left|\tilde{C}>=h_{R}\right| \tilde{C}>, \quad \bar{L}_{0}\left|\tilde{D}>=h_{R}\right| \tilde{D}>+\mid \tilde{C}>.
$$

Here the two-point functions of these operators take the forms

$$
<\tilde{C}(\bar{x}) \tilde{C}(0)>=0, \quad<\tilde{C}(\bar{x}) \tilde{D}(0)>=\frac{\bar{c}}{\bar{x}^{2 h_{R}}}, \quad<\tilde{D}(\bar{x}) \tilde{D}(0)>=\frac{1}{\bar{x}^{2 h_{R}}}[\bar{d}-2 \bar{c} \log (\bar{x})] .
$$

On the other hand, its momentum space two-point functions take the form

$$
\overline{\mathcal{D}}^{\tilde{D} \tilde{C}}\left(p_{-}\right) \propto \Gamma\left(h_{R}+i \frac{p_{-}}{2 \pi T_{R}}\right) \Gamma\left(h_{R}-i \frac{p_{-}}{2 \pi T_{R}}\right)
$$

where $h_{R}=(m-2) / 2, p_{-}=(\omega-k) / 2$, and $T_{R}=1 / 2 \pi$. Confining the poles in Eq. (75) to the lower half-plane, one finds the other set of simple poles

$$
\omega_{R}=k-2 i\left(n+h_{R}\right) .
$$

This set of poles characterizes the decay of the perturbation on the $\mathrm{LCFT}_{R}$ side.

Finally, we wish to mention that the above AdS/LCFT construction is closely related to the spin-2 case since the bulk-boundary correspondence is irrelevant to the higher spin $N$. Therefore, we suggest that the spin-2 computations of 2- and 3-point correlators [21], and the 1-loop partition function [22] may be helpful to calculate those of spin-3. 


\section{Discussions}

Using the operator method, we have constructed the logarithmic quasinormal modes of a traceless spin-3 field around the BTZ black hole at the critical point of the spin-3 TMG. The quasinormal frequencies are given by $\omega_{L / R}^{n}=\mp k-2 i n$. The positive integer " $n$ " implies that the BTZ black hole is stable against the spin-3 perturbations because there is no exponentially growing modes like $e^{2 n t}$. We note that these quasinormal frequencies are the same as those of the spin-2 graviton.

The logarithmic quasinormal modes depending $y(t, \rho)=-(t+\ln \sinh \rho)$ reflect that the linearized equation (26) is a third-order differential equation. The presence of the log spin3 mode may induce the instability of the BTZ black hole spacetimes and the non-chiral nature of the spin-3 field coupled to topologically massive gravity. As far as the instability issue concerned, we have to pay attention to the log spin-2 case of [11] where the BrownHenneaux boundary conditions are relaxed to allow metric fluctuations to grow linearly in $\rho$ at infinity. Also, if one relaxes the boundary condition to allow log-modes (whose presence makes the theory non-chiral) [23, 24], one has the well-defined logarithmic quasinormal modes. Moreover, even though there is a linearized instability, logarithmic excitations always obey log-boundary conditions, but not the Brown-Henneaux boundary conditions [25]. At this stage, it is important to mention that the $y(t, \rho)$-dependence of the spin-3 quasinormal modes is necessary to reproduce the double poles in Eq. (69). In this direction, we wish to note that the appearance of a simple pole in the retarded Green function is closely related to quasinormal modes [20], while the appearance of a double pole in the retarded Green function reflects logarithmic quasinormal modes [14]. Hence, the instability due to $y(t, \rho)$ is not considered as an obstacle to the interpretation of log spin-3 quasinormal modes.

Finally, we have established the bulk-boundary correspondence by introducing two sets of operators $(D, C)$ for the $\operatorname{LCFT}_{L}$ and $(\tilde{D}, \tilde{C})$ for the $\mathrm{LCFT}_{R}$. We could read off the logarithmic quasinormal frequencies of $\omega_{L / R}^{n}$ from the locations of retarded green function in momentum space $\mathcal{D}^{D C}\left(p_{+}\right)$and $\overline{\mathcal{D}}^{\tilde{D} \tilde{C}}\left(p_{-}\right)$[20]. These are $\omega_{L / R}^{L C F T, n}=\mp k-2 i\left(n+h_{L / R}\right)$ where $h_{L / R}(m)=(m-2) / 2$ is zero for $m=2$, leading to $\omega_{L / R}^{n}=\omega_{L / R}^{L C F T, n}$. It dictates that using the AdS/LCFT correspondence [14, the logarithmic quasinormal frequencies could be obtained from the $\mathrm{LCFT}_{L / R}$ sides.

\section{Acknowledgement}

Two of us (Y. S. Myung and Y.-W. Kim) were supported by the National Research Foundation of Korea (NRF) grant funded by the Korea government (MEST) (Grant No.2011- 
0027293). Y.-J. Park was partially supported by the National Research Foundation of Korea (NRF) Grant funded by the Korea government (MEST) through the Center for Quantum Spacetime (CQUeST) of Sogang University with Grant No. 2005-0049409, and was also supported by World Class University program funded by the Ministry of Education, Science and Technology through the National Research Foundation of Korea (Grant No. R31-20002). 


\section{Appendix: Full forms of the descendants}

\section{A1. The second descendants of the left-logarithmic modes}

The full form of the components of the second descendants (52)-(54) are

$$
\begin{aligned}
& f_{\text {uuv }}^{L(2)}=14-4 i k+2\left(12-7 i k-k^{2}\right) y(t, \rho), \\
& f_{\text {uu } \rho}^{L(2)}=2 f_{\text {uuv }}^{L(2)}=2\left(14-4 i k+2\left(12-7 i k-k^{2}\right) y(t, \rho)\right), \\
& f_{\text {uvv }}^{L(2)}=40-29 i k-5 k^{2}+\left(26-11 i k-k^{2}\right) \cosh 2 \rho \\
& +2\left(24-26 i k-9 k^{2}+i k^{3}+\left(12-7 i k-k^{2}\right) \cosh 2 \rho\right) y(t, \rho), \\
& f_{u v \rho}^{L(2)}=2\left(40-29 i k-5 k^{2}+\left(40-15 i k-k^{2}\right) \cosh 2 \rho\right. \\
& \left.+2\left(24-26 i k-9 k^{2}+i k^{3}+\left(24-14 i k-2 k^{2}\right) \cosh 2 \rho\right) y(t, \rho)\right), \\
& f_{u \rho \rho}^{L(2)}=4\left(40-29 i k-5 k^{2}+\left(54-19 i k-k^{2}\right) \cosh 2 \rho\right. \\
& \left.+2\left(24-26 i k-9 k^{2}+i k^{3}+\left(36-21 i k-3 k^{2}\right) \cosh 2 \rho\right) y(t, \rho)\right), \\
& f_{v v v}^{L(2)}=\frac{1}{8}\left(452-517 i k-195 k^{2}+24 i k^{3}+4\left(88-81 i k-23 k^{2}+2 i k^{3}\right) \cosh 2 \rho\right. \\
& \left.+\left(12-7 i k-k^{2}\right) \cosh 4 \rho\right) \\
& +\left(48-76 i k-44 k^{2}+11 i k^{3}+k^{4}+\left(24-26 i k-9 k^{2}+i k^{3}\right) \cosh 2 \rho\right) y(t, \rho), \\
& f_{v v \rho}^{L(2)}=\frac{1}{4}\left(584-569 i k-199 k^{2}+24 i k^{3}+4\left(168-139 i k-33 k^{2}+2 i k^{3}\right) \cosh 2 \rho\right. \\
& \left.+\left(88-43 i k-5 k^{2}\right) \cosh 4 \rho\right) \\
& +\left(132-173 i k-91 k^{2}+22 i k^{3}+2 k^{4}+\left(144-156 i k-54 k^{2}+6 i k^{3}\right) \cosh 2 \rho\right. \\
& \left.+\left(12-7 i k-k^{2}\right) \cosh 4 \rho\right) y(t, \rho) \text {, } \\
& f_{v \rho \rho}^{L(2)}=\frac{1}{2}\left(772-637 i k-203 k^{2}+24 i k^{3}+4\left(248-197 i k-43 k^{2}+2 i k^{3}\right) \cosh 2 \rho\right. \\
& \left.+\left(220-95 i k-9 k^{2}\right) \cosh 4 \rho\right) \\
& +4\left(96-104 i k-48 k^{2}+11 i k^{3}+k^{4}+\left(120-130 i k-45 k^{2}+5 i k^{3}\right) \cosh 2 \rho\right. \\
& \left.+\left(24-14 i k-2 k^{3}\right) \cosh 4 \rho\right) y(t, \rho) \text {, } \\
& f_{\rho \rho \rho}^{L(2)}=\left(1016-721 i k-207 k^{2}+24 i k^{3}+4\left(328-255 i k-53 k^{2}+2 i k^{3}\right) \cosh 2 \rho\right. \\
& \left.+\left(408-163 i k-13 k^{2}\right) \cosh 4 \rho\right) \\
& +4\left(276-257 i k-103 k^{2}+22 i k^{3}+2 k^{4}\right. \\
& +\left(336-364 i k-126 k^{2}+14 i k^{3}\right) \cosh 2 \rho \\
& \left.+\left(108-63 i k-9 k^{2}\right) \cosh 4 \rho\right) y(t, \rho) \text {. }
\end{aligned}
$$




\section{A2. The third descendants of the left-logarithmic modes}

The full form of the components of the third descendants (56)-(58) are

$$
\begin{aligned}
& f_{\text {uuu }}^{L(3)}=6\left(47-24 i k-3 k^{2}+\left(60-47 i k-12 k^{2}+i k^{3}\right) y(t, \rho)\right), \\
& f_{\text {uuv }}^{L(3)}=3\left(342-285 i k-78 k^{2}+7 i k^{3}+\left(248-143 i k-24 k^{2}+i k^{3}\right) \cosh 2 \rho\right. \\
& \left.+2\left(180-201 i k-83 k^{2}+15 i k^{3}+k^{4}+2\left(60-47 i k-12 k^{2}+i k^{3}\right) \cosh 2 \rho\right) y(t, \rho)\right) \text {, } \\
& f_{\text {uu }}^{L(3)}=6\left(342-285 i k-78 k^{2}+7 i k^{3}+\left(342-191 i k-30 k^{2}+i k^{3}\right) \cosh 2 \rho\right. \\
& \left.+2\left(180-201 i k-83 k^{2}+15 i k^{3}+k^{4}+3\left(60-48 i k-12 k^{2}+i k^{3}\right) \cosh 2 \rho\right) y(t, \rho)\right) \text {, } \\
& f_{u v v}^{L(3)}=\frac{3}{8}\left(5714-6161 i k-2522 k^{2}+463 i k^{3}+32 k^{4}\right. \\
& +4\left(1386-1257 i k-400 k^{2}+51 i k^{3}+2 k^{4}\right) \cosh 2 \rho \\
& +\left(582-379 i k-78 k^{2}+5 i k^{3}\right) \cosh 4 \rho \\
& +\left(2\left(2460-3367 i k-1860 k^{2}+517 i k^{3}+72 k^{4}-4 i k^{5}\right)\right. \\
& +24\left(180-201 i k-83 k^{2}+15 i k^{3}+k^{4}\right) \cosh 2 \rho \\
& \left.\left.+6\left(60-47 i k-12 k^{2}+i k^{3}\right) \cosh 4 \rho\right) y(t, \rho)\right) \text {, } \\
& f_{u v \rho}^{L(3)}=\frac{3}{4}\left(6894-6829 i k-2630 k^{2}+467 i k^{3}+32 k^{4}\right. \\
& +4\left(2070-1827 i k-556 k^{2}+65 i k^{3}+2 k^{4}\right) \cosh 2 \rho+9\left(154-95 i k-18 k^{2}+i k^{3}\right) \cosh 4 \rho \\
& +\left(2\left(3060-3837 i k-1980 k^{2}+527 i k^{3}+72 k^{4}-4 i k^{5}\right)\right. \\
& +40\left(180-201 i k-83 k^{2}+15 i k^{3}+k^{4}\right) \cosh 2 \rho \\
& \left.\left.+18\left(60-47 i k-12 k^{2}+i k^{3}\right) \cosh 4 \rho\right) y(t, \rho)\right) \text {, } \\
& f_{u \rho \rho}^{L(3)}=\frac{3}{2}\left(8450-7689 i k-2762 k^{2}+471 i k^{3}+32 k^{4}\right. \\
& +4\left(2754-2397 i k-712 k^{2}+79 i k^{3}+2 k^{4}\right) \cosh 2 \rho \\
& +\left(2566-1523 i k-270 k^{2}+13 i k^{3}\right) \cosh 4 \rho \\
& +\left(2\left(3900-4495 i k-2148 k^{2}+541 i k^{3}+72 k^{4}-4 i k^{5}\right)\right. \\
& +56\left(180-201 i k-83 k^{2}+15 i k^{3}+k^{4}\right) \cosh 2 \rho \\
& \left.\left.+38\left(60-47 i k-12 k^{2}+i k^{3}\right) \cosh 4 \rho\right) y(t, \rho)\right) \text {, } \\
& f_{v v v}^{L(3)}=\frac{1}{16}\left(6\left(7014-9724 i k-5417 k^{2}+1516 i k^{3}+213 k^{4}-12 i k^{5}\right)\right. \\
& +3\left(14268-17683 i k-8476 k^{2}+1941 i k^{3}+208 k^{4}-8 i k^{5}\right) \cosh 2 \rho \\
& +6\left(882-888 i k-327 k^{2}+52 i k^{3}+3 k^{4}\right) \cosh 4 \rho+\left(60-47 i k-12 k^{2}+i k^{3}\right) \cosh 6 \rho \\
& +\left(4\left(7380-12561 i k-8947 k^{2}+3411 i k^{3}+733 k^{4}-84 i k^{5}-4 k^{6}\right)\right. \\
& +48\left(540-783 i k-450 k^{2}+128 i k^{3}+18 k^{4}-i k^{5}\right) \cosh 2 \rho \\
& \left.\left.+\quad 12\left(180-201 i k-83 k^{2}+15 i k^{3}+k^{4}\right) \cosh 4 \rho\right) y(t, \rho)\right) \text {, }
\end{aligned}
$$




$$
\begin{aligned}
& f_{v v \rho}^{L(3)}=\frac{1}{4}\left(3\left(10470-12808 i k-6373 k^{2}+1632 i k^{3}+217 k^{4}-12 i k^{5}\right)\right. \\
& +3\left(13293-15287 i k-6817 k^{2}+1437 i k^{3}+136 k^{4}-4 i k^{5}\right) \cosh 2 \rho \\
& +3\left(2970-2832 i k-971 k^{2}+140 i k^{3}+7 k^{4}\right) \cosh 4 \rho \\
& +\left(441-307 i k-69 k^{2}+5 i k^{3}\right) \cosh 6 \rho \\
& +\left(2\left(11700-17385 i k-10939 k^{2}+3771 i k^{3}+757 k^{4}-84 i k^{5}-4 k^{6}\right)\right. \\
& +3\left(9540-13233 i k-7380 k^{2}+2063 i k^{3}+288 k^{4}-16 i k^{5}\right) \cosh 2 \rho \\
& +30\left(180-201 i k-83 k^{2}+15 i k^{3}+k^{4}\right) \cosh 4 \rho \\
& \left.\left.+3\left(60-47 i k-12 k^{2}+i k^{3}\right) \cosh 6 \rho\right) y(t, \rho)\right) \text {, } \\
& f_{v \rho \rho}^{L(3)}=\frac{1}{4}\left(6\left(15294-17032 i k-7641 k^{2}+1776 i k^{3}+221 k^{4}-12 i k^{5}\right)\right. \\
& +3\left(42256-45373 i k-19104 k^{2}+3819 i k^{3}+336 k^{4}-8 i k^{5}\right) \cosh 2 \rho \\
& +6\left(6426-5916 i k-1927 k^{2}+256 i k^{3}+11 k^{4}\right) \cosh 4 \rho \\
& +\left(3552-2321 i k-480 k^{2}+31 i k^{3}\right) \cosh 6 \rho \\
& +\left(4\left(18180-24621 i k-13927 k^{2}+4311 i k^{3}+793 k^{4}-84 i k^{5}-4 k^{6}\right)\right. \\
& +12\left(8220-10759 i k-5748 k^{2}+1565 i k^{3}+216 k^{4}-12 i k^{5}\right) \cosh 2 \rho \\
& +156\left(180-201 i k-83 k^{2}+15 i k^{3}+k^{4}\right) \cosh 4 \rho \\
& \left.\left.+36\left(60-47 i k-12 k^{2}+i k^{3}\right) \cosh 6 \rho\right) y(t, \rho)\right) \text {, } \\
& f_{\rho \rho \rho}^{L(3)}=\left(3\left(21486-22396 i k-9221 k^{2}+1948 i k^{3}+225 k^{4}-12 i k^{5}\right)\right. \\
& +3\left(31203-31328 i k-12479 k^{2}+2388 i k^{3}+200 k^{4}-4 i k^{5}\right) \cosh 2 \rho \\
& +15\left(2250-2028 i k-639 k^{2}+80 i k^{3}+3 k^{4}\right) \cosh 4 \rho \\
& +\left(4599-2872 i k-555 k^{2}+32 i k^{3}\right) \cosh 6 \rho \\
& +\left(2\left(26820-34269 i k-17911 k^{2}+5031 i k^{3}+841 k^{4}-84 i k^{5}-4 k^{6}\right)\right. \\
& +3\left(25740-31683 i k-16092 k^{2}+4237 i k^{3}+576 k^{4}-32 i k^{5}\right) \cosh 2 \rho \\
& +\quad 150\left(180-201 i k-83 k^{2}+15 i k^{3}+k^{4}\right) \cosh 4 \rho \\
& \left.\left.+57\left(60-47 i k-12 k^{2}+i k^{3}\right) \cosh 6 \rho\right) y(t, \rho)\right) \text {. }
\end{aligned}
$$

\section{A3. The fourth descendent of the left-logarithmic modes}

Here we summarize the fourth descendent of the left-logarithmic modes for the s-mode $(k=0$ case)

$$
\Phi_{\rho \mu \nu}^{L(4), n e w}(u, v, \rho)=\left(\bar{L}_{-1} L_{-1}\right)^{4} \Phi_{\rho \mu \nu}^{L, n e w}(u, v, \rho) .
$$


Explicitly, we have

$$
\begin{aligned}
\left(\bar{L}_{-1} L_{-1}\right)^{4} \Phi_{u \mu \nu}^{L, \text { new }}(u, v, \rho) & =\frac{e^{-8 t}}{\sinh ^{8} \rho}\left(\begin{array}{ccc}
f_{u u u}^{L(4)} & f_{u u v}^{L(4)} & \frac{f_{u u \rho}^{L(4)}}{\sinh 2 \rho} \\
f_{u u v}^{L(4)} & f_{u v v}^{L(4)} & \frac{f_{u v \rho}^{L(4)}}{\sinh 2 \rho} \\
\frac{f_{u u \rho}^{L(4)}}{\sinh 2 \rho} & \frac{f_{u v \rho}^{L(4)}}{\sinh 2 \rho} & \frac{f_{u \rho \rho}^{L(4)}}{\sinh ^{2} 2 \rho}
\end{array}\right)_{\mu \nu}, \\
\left(\bar{L}_{-1} L_{-1}\right)^{4} \Phi_{v \mu \nu}^{L, \text { new }}(u, v, \rho) & =\frac{e^{-8 t}}{\sinh ^{8} \rho}\left(\begin{array}{ccc}
f_{u u v}^{L(4)} & f_{u v v}^{L(4)} & \frac{f_{u v \rho}^{L(4)}}{\sinh 2 \rho} \\
f_{u v v}^{L(4)} & f_{v v v}^{L(4)} & \frac{f_{v v \rho}^{L(4)}}{\sinh ^{L} 2 \rho} \\
\frac{f_{u v \rho}^{L(4)}}{\sinh 2 \rho} & \frac{f_{v v \rho}^{L(4)}}{\sinh 2 \rho} & \frac{f_{v \rho \rho}^{L(4)}}{\sinh ^{2} 2 \rho}
\end{array}\right)_{\mu \nu}, \\
\left(\bar{L}_{-1} L_{-1}\right)^{4} \Phi_{\rho \mu \nu}^{L, n e w}(u, v, \rho) & =\frac{e^{-8 t}}{\sinh ^{8} \rho}\left(\begin{array}{ccc}
\frac{f_{u u \rho}^{L(4)}}{\sinh 2 \rho} & \frac{f_{u v \rho}^{L(4)}}{\sinh 2 \rho} & \frac{f_{u \rho \rho}^{L(4)}}{\sinh ^{2} 2 \rho} \\
\frac{f_{u v \rho}^{L(4)}}{\sinh 2 \rho} & \frac{f_{v v \rho}^{L(4)}}{\sinh 2 \rho} & \frac{f_{v \rho \rho}^{L(4)}}{\sinh ^{2} 2 \rho} \\
\frac{f_{u \rho \rho}^{L(4)}}{\sinh ^{2} 2 \rho} & \frac{f_{v \rho \rho}^{L(4)}}{\sinh ^{2} 2 \rho} & \frac{f_{\rho \rho \rho}^{L} 2 \rho}{\sinh ^{3} 2 \rho}
\end{array}\right)_{\mu \nu},
\end{aligned}
$$

where

$$
\begin{aligned}
f_{u u u}^{L(4)} & =432(86+67 \cosh 2 \rho)+8640(4+3 \cosh 2 \rho) y(t, \rho), \\
f_{u u v}^{L(4)} & =432(239+255 \cosh 2 \rho+35 \cosh 4 \rho)+2160(39+40 \cosh 2 \rho+5 \cosh 4 \rho) y(t, \rho), \\
f_{u u \rho}^{L(4)} & =432(1+\cosh 2 \rho)(427+255 \cosh 2 \rho+40(9+5 \cosh 2 \rho) y(t, \rho)) \\
f_{u v v}^{L(4)} & =36(5050+5955 \cosh 2 \rho+1194 \cosh 4 \rho+61 \cosh 6 \rho) \\
& +1080(120+135 \cosh 2 \rho+24 \cosh 4 \rho+\cosh 6 \rho) y(t, \rho) \\
f_{u v \rho}^{L(4)} & =(1+\cosh 2 \rho)(144(2385+2068 \cosh 2 \rho+199 \cosh 4 \rho) \\
& +17280(15+12 \cosh 2 \rho+\cosh 4 \rho) y(t, \rho)) \\
f_{u \rho \rho}^{L(4)} & =2(1+\cosh 2 \rho)(144(2925+3292 \cosh 2 \rho+481 \cosh 4 \rho) \\
& +4320(75+82 \cosh 2 \rho+11 \cosh 4 \rho) y(t, \rho)) \\
f_{v v v}^{L(4)} & =\frac{9}{8}(186125+223320 \cosh 2 \rho+47268 \cosh 4 \rho+2792 \cosh 6 \rho+15 \cosh 8 \rho) \\
& +1080(120+135 \cosh 2 \rho+24 \cosh 4 \rho+\cosh 6 \rho) y(t, \rho) \\
f_{v v \rho}^{L(4)} & =(1+\cosh 2 \rho)\left(\frac{9}{2}(101650+97875 \cosh 2 \rho+13086 \cosh 4 \rho+349 \cosh 6 \rho)\right. \\
& +540(570+495 \cosh 2 \rho+54 \cosh 4 \rho+\cosh 6 \rho) y(t, \rho)) \\
f_{v \rho \rho}^{L(4)} & =2(1+\cosh 2 \rho)\left(\frac{9}{2}(145670+177121 \cosh 2 \rho+37066 \cosh 4 \rho+1967 \cosh 6 \rho)\right. \\
& +4320(105+123 \cosh 2 \rho+23 \cosh 4 \rho+\cosh 6 \rho) y(t, \rho)) \\
f_{\rho \rho \rho}^{L(4)} & =2(1+\cosh 2 \rho)^{2}(18(71495+65828 \cosh 2 \rho+6717 \cosh 4 \rho) \\
& +6480(145+124 \cosh 2 \rho+11 \cosh 4 \rho) y(t, \rho))
\end{aligned}
$$




\section{B. The asymptotic form of the left-moving noncritical modes}

The solution of the first order equation (36) is given by

$$
\Phi_{\rho \mu \nu}^{L}(u, v, \rho)=e^{-(\mu-1)(u+v)}(\sinh \rho)^{-2(\mu-1)} F_{\rho \mu \nu}^{L},
$$

where $\mu>1$. From this and the corresponding descendent solutions, we find that the asymptotic forms are given as follows

$$
\begin{aligned}
\Phi_{u \mu \nu}^{L, \infty}(u, v, \rho) & \sim e^{-2(\mu-1) t}\left(\begin{array}{ccc}
0 & 0 & 0 \\
0 & 0 & 0 \\
0 & 0 & 0
\end{array}\right)_{\mu \nu}, \\
\Phi_{v \mu \nu}^{L, \infty}(u, v, \rho) & \sim e^{-2(\mu-1) t}\left(\begin{array}{ccc}
0 & 0 & 0 \\
0 & e^{-2(\mu-1) \rho} & e^{-2 \mu \rho} \\
0 & e^{-2 \mu \rho} & e^{-2(\mu+1) \rho}
\end{array}\right)_{\mu \nu}, \\
\Phi_{\rho \mu \nu}^{L, \infty}(u, v, \rho) & \sim e^{-2(\mu-1) t}\left(\begin{array}{ccc}
0 & 0 & 0 \\
0 & e^{-2 \mu \rho} & e^{-2(\mu+1) \rho} \\
0 & e^{-2(\mu+1) \rho} & e^{-2(\mu+2) \rho}
\end{array}\right)_{\mu \nu} .
\end{aligned}
$$

For the first descendent solutions, we have

$$
\begin{gathered}
\Phi_{u \mu \nu}^{L(1), \infty}(u, v, \rho) \sim e^{-2 \mu t}\left(\begin{array}{ccc}
0 & 0 & 0 \\
0 & e^{-2 \mu \rho} & e^{-2(\mu+1) \rho} \\
0 & e^{-2(\mu+1) \rho} & e^{-2(\mu+2) \rho}
\end{array}\right)_{\mu \nu}, \\
\Phi_{v \mu \nu}^{L(1), \infty}(u, v, \rho) \sim e^{-2 \mu t}\left(\begin{array}{ccc}
0 & e^{-2 \mu \rho} & e^{-2(\mu+1) \rho} \\
e^{-2 \mu \rho} & e^{-2(\mu-1) \rho} & e^{-2 \mu \rho} \\
e^{-2(\mu+1) \rho} & e^{-2 \mu \rho} & e^{-2(\mu+1) \rho}
\end{array}\right)_{\mu \nu}, \\
\Phi_{\rho \mu \nu}^{L(1), \infty}(u, v, \rho) \sim e^{-2 \mu t}\left(\begin{array}{ccc}
0 & e^{-2(\mu+1) \rho} & e^{-2(\mu+2) \rho} \\
e^{-2(\mu+1) \rho} & e^{-2 \mu \rho} & e^{-2(\mu+1) \rho} \\
e^{-2(\mu+2) \rho} & e^{-2(\mu+1) \rho} & e^{-2(\mu+2) \rho}
\end{array}\right)_{\mu \nu} .
\end{gathered}
$$


For the second descendent solutions, we have

$$
\begin{aligned}
& \Phi_{u \mu \nu}^{L(2), \infty}(u, v, \rho) \sim e^{-2(\mu+1) t}\left(\begin{array}{ccc}
0 & e^{-2(\mu+1) \rho} & e^{-2(\mu+2) \rho} \\
e^{-2(\mu+1) \rho} & e^{-2 \mu \rho} & e^{-2(\mu+1) \rho} \\
e^{-2(\mu+2) \rho} & e^{-2(\mu+1) \rho} & e^{-2(\mu+2) \rho}
\end{array}\right)_{\mu \nu}, \\
& \Phi_{v \mu \nu}^{L(2), \infty}(u, v, \rho) \sim e^{-2(\mu+1) t}\left(\begin{array}{ccc}
e^{-2(\mu+1) \rho} & e^{-2 \mu \rho} & e^{-2(\mu+1) \rho} \\
e^{-2 \mu \rho} & e^{-2(\mu-1) \rho} & e^{-2 \mu \rho} \\
e^{-2(\mu+1) \rho} & e^{-2 \mu \rho} & e^{-2(\mu+1) \rho}
\end{array}\right)_{\mu \nu}, \\
& \Phi_{\rho \mu \nu}^{L(2), \infty}(u, v, \rho) \sim e^{-2(\mu+1) t}\left(\begin{array}{ccc}
e^{-2(\mu+2) \rho} & e^{-2(\mu+1) \rho} & e^{-2(\mu+2) \rho} \\
e^{-2(\mu+1) \rho} & e^{-2 \mu \rho} & e^{-2(\mu+1) \rho} \\
e^{-2(\mu+2) \rho} & e^{-2(\mu+1) \rho} & e^{-2(\mu+2) \rho}
\end{array}\right)_{\mu \nu} .
\end{aligned}
$$

For the third descendent solutions, we have

$$
\begin{aligned}
& \Phi_{u \mu \nu}^{L(3), \infty}(u, v, \rho) \sim e^{-2(\mu+2) t}\left(\begin{array}{ccc}
e^{-2(\mu+2) \rho} & e^{-2(\mu+1) \rho} & e^{-2(\mu+2) \rho} \\
e^{-2(\mu+1) \rho} & e^{-2 \mu \rho} & e^{-2(\mu+1) \rho} \\
e^{-2(\mu+2) \rho} & e^{-2(\mu+1) \rho} & e^{-2(\mu+2) \rho}
\end{array}\right)_{\mu \nu}, \\
& \Phi_{v \mu \nu}^{L(3), \infty}(u, v, \rho) \sim e^{-2(\mu+2) t}\left(\begin{array}{ccc}
e^{-2(\mu+1) \rho} & e^{-2 \mu \rho} & e^{-2(\mu+1) \rho} \\
e^{-2 \mu \rho} & e^{-2(\mu-1) \rho} & e^{-2 \mu \rho} \\
e^{-2(\mu+1) \rho} & e^{-2 \mu \rho} & e^{-2(\mu+1) \rho}
\end{array}\right)_{\mu \nu}, \\
& \Phi_{\rho \mu \nu}^{L(3), \infty}(u, v, \rho) \sim e^{-2(\mu+2) t}\left(\begin{array}{ccc}
e^{-2(\mu+2) \rho} & e^{-2(\mu+1) \rho} & e^{-2(\mu+2) \rho} \\
e^{-2(\mu+1) \rho} & e^{-2 \mu \rho} & e^{-2(\mu+1) \rho} \\
e^{-2(\mu+2) \rho} & e^{-2(\mu+1) \rho} & e^{-2(\mu+2) \rho}
\end{array}\right)_{\mu \nu} .
\end{aligned}
$$

We note here that the fourth descendent of the solution has been calculated for the s-mode ( $k=0$ case), without loss of generosity. As a result, we have confirmed that they are the same with the asymptotic form of the third descendent Eq. (88).

\section{References}

[1] M. Henneaux and S. -J. Rey, JHEP 1012 (2010) 007 [arXiv:1008.4579 [hep-th]].

[2] A. Campoleoni, S. Fredenhagen, S. Pfenninger and S. Theisen, JHEP 1011 (2010) 007 arXiv:1008.4744 [hep-th]].

[3] B. Chen, J. Long and J.-B. Wu, Phys. Lett. B 705 (2011) 513 [arXiv:1106.5141 [hep-th]]. 
[4] A. Bagchi, S. Lal, A. Saha and B. Sahoo, JHEP 1110 (2011) 150 arXiv:1107.0915 [hep-th]].

[5] W. Li, W. Song and A. Strominger, JHEP 0804 (2008) 082 [arXiv:0801.4566 [hep-th]].

[6] S. Datta and J. R. David, JHEP 1203 (2012) 079 [arXiv:1112.4619 [hep-th]].

[7] I. Sachs and S. N. Solodukhin, JHEP 0808 (2008) 003 [arXiv:0806.1788 [hep-th]].

[8] D. Birmingham, S. Mokhtari and I. Sachs, Phys. Rev. D 82 (2010) 124059 arXiv:1006.5524 [hep-th]].

[9] Y. S. Myung, Y. W. Kim, T. Moon and Y. J. Park, Phys. Rev. D 84 (2011) 024044 arXiv:1105.4205 [hep-th]].

[10] Y. S. Myung, Y. -W. Kim and Y. -J. Park, Phys. Rev. D 86 (2012) 084007 arXiv:1201.3964 [hep-th]].

[11] D. Grumiller and N. Johansson, JHEP 0807 (2008) 134 arXiv:0805.2610 [hep-th]].

[12] G. Giribet, M. Kleban and M. Porrati, JHEP 0810, 045 (2008) arXiv:0807.4703 [hepth]].

[13] Y. S. Myung, Phys. Lett. B 670 (2008) 220 [arXiv:0808.1942 [hep-th]].

[14] I. Sachs, JHEP 0809 (2008) 073 [arXiv:0807.1844 [hep-th]].

[15] F. Belgiorno, S. L. Cacciatori, F. D. Piazza and O. F. Piattella, J. Phys. A 44 (2011) 025202 arXiv:1007.4439 [math-ph]].

[16] J. D. Brown and M. Henneaux, Commun. Math. Phys. 104, 207 (1986).

[17] M. Flohr, Int. J. Mod. Phys. A 18 (2003) 4497 arXiv:hep-th/0111228.

[18] Y. S. Myung and H. W. Lee, JHEP 9910 (1999) 009 [arXiv:hep-th/9904056].

[19] A. Lewis, Phys. Lett. B 480 (2000) 348 arXiv:hep-th/9911163.

[20] D. Birmingham, I. Sachs and S. N. Solodukhin, Phys. Rev. Lett. 88 (2002) 151301 hep-th/0112055.

[21] D. Grumiller and I. Sachs, JHEP 1003, 012 (2010) arXiv:0910.5241 [hep-th]]. 
[22] M. R. Gaberdiel, D. Grumiller and D. Vassilevich, JHEP 1011, 094 (2010) arXiv:1007.5189 [hep-th]].

[23] D. Grumiller and N. Johansson, Int. J. Mod. Phys. D 17, 2367 (2009) arXiv:0808.2575 [hep-th]].

[24] M. Henneaux, C. Martinez and R. Troncoso, Phys. Rev. D 79, 081502 (2009) arXiv:0901.2874 [hep-th]].

[25] A. Maloney, W. Song and A. Strominger, Phys. Rev. D 81, 064007 (2010) arXiv:0903.4573 [hep-th]]. 